PACS $61.72,72.20$

\title{
Electron-electron drag in crystals with multivalley band
}

\author{
I.I. Boiko \\ V. Lashkaryov Institute of Semiconductor Physics, NAS of Ukraine, 45, prospect Nauky, 03028 Kyiv, Ukraine \\ E-mail: igorboiko@yandex.ru; phone: (38-044)236-5422
}

\begin{abstract}
Mobility of electrons in multivalley bands of Si and Ge is considered with due regard for intervalley drag. It is shown that drag sufficiently diminishes electron mobility at low temperatures. This effect is clearer pronounced in germanium than in silicon.
\end{abstract}

Keywords: quantum kinetic equation, mobility, intervalley drag.

Manuscript received 03.04.09; accepted for publication 14.05.09; published online 15.05.09.

\section{Introduction}

In absence of external electric field, electron-electron interaction in uniform medium is usually considered as a principal mechanism of electron gas thermalization and formation of a dielectric function for the studied system. In crystals with simple band electron-electron scattering, the latter does not change the total momentum of carriers and, therefore, has no direct, independent contribution to conductivity (see [1-3]). For non-equilibrium electron gas that is represented by only one group, the mentioned interaction manifests itself mainly as a mechanism of formation of symmetrical part for the distribution function. But in the case when mobile carriers belong to different groups with rare transitions between these groups, quite specific contribution of $e-e$-interaction to conductivity appears. This effect is related with a difference of drift velocities for different groups and manifests as intergroup drag. The most interesting objects for consideration of the drag are electrons in multivalley semiconductor with equivalent anisotropic valleys (all groups have the same population and that favours to effect). In details, we will examine the intervalley drag in $n$-Si and $n$-Ge. This phenomenon better displays in the region of sufficiently low temperatures where Coulomb scattering is not damped by collisions with phonons.

\section{Quantum kinetic equation}

Let us consider here a uniform crystal in constant uniform electric field $\vec{E}$. In this case, the stationary quantum kinetic equation for a distribution function $f_{\vec{k}}^{(a)}$ for carriers from $a$-group can be presented in the following form (see, for instance, Refs [4-7]):

$$
\begin{aligned}
& \frac{e_{a}}{\hbar} \vec{E} \frac{\partial f_{\vec{k}}^{(a)}}{\partial \vec{k}}=S t f_{\vec{k}}^{(a)}=S t_{e-I} f_{\vec{k}}^{(a)}+S t_{e-p h} f_{\vec{k}}^{(a)}+ \\
& +\sum_{b=1}^{M} S t_{a-b} f_{\vec{k}}^{(a)}(a, b=1,2, \ldots, M) .
\end{aligned}
$$

Here, $M=6$ for $n-\mathrm{Si}$, and $M=4$ for $n-\mathrm{Ge}$. In Eq. (1) we suppose the same electric charge for mobile carriers from different groups. The collision integral in the right part of this equation involves scattering of $a$ carriers by charged impurities ( $e-I$ process), phonons ( $e$ $p h$ process) and band carriers belonging to all the considered groups ( $a-b$ process). Below, we restrict our consideration with quasi-elastic scattering by longitudinal acoustic phonons. Intervalley transitions are not taken in consideration. We also assume that intergroups transitions can be neglected in comparison with direct Coulomb scattering by band carriers.

The screening dielectric function $\varepsilon(\omega, q)$ for quasielastic collisions has the form

$\varepsilon(\omega, \vec{q})=\varepsilon_{L}+\Delta \varepsilon_{e}(\omega=0, \vec{q})$,

where $\varepsilon_{L}$ is the dielectric constant of crystal lattice and $\Delta \varepsilon_{e}(\omega, \vec{q})$ is contribution of band electrons to total dielectric function. Farther we accept the following designation:

$$
\Delta \varepsilon(0, \vec{q})=\varepsilon_{L} q_{0}^{2}(\vec{q}) / q^{2} .
$$

Using the simplified model (2) and form (3) for the screening dielectric function, we write (see Ref. [4]) 


$$
\begin{aligned}
& S t_{a-b} f_{\vec{k}}^{(a)}=\frac{e^{4}}{\pi^{3} \hbar} \int d^{3} \vec{k}^{\prime} \int d^{3} \vec{q} \frac{\delta\left(\varepsilon_{\vec{k}}^{(a)}-\varepsilon_{\vec{k}-\vec{q}}^{(a)}+\varepsilon_{\vec{k}^{\prime}-\vec{q}}^{(b)}-\varepsilon_{\vec{k}^{\prime}}^{(b)}\right)}{\varepsilon_{L}^{2}\left[q^{2}+q_{0}^{2}(\vec{q})\right]^{2}} \times \\
& \times\left[f_{\vec{k}-\vec{q}}^{(a)}\left(1-f_{\vec{k}}^{(a)}\right) f_{\vec{k}^{\prime}}^{(b)}\left(1-f_{\vec{k}^{\prime}-\vec{q}}^{(b)}\right)-\right. \\
& \left.-f_{\vec{k}^{\prime}-\vec{q}}^{(b)}\left(1-f_{\vec{k}^{\prime}}^{(b)}\right) f_{\vec{k}}^{(a)}\left(1-f_{\vec{k}-\vec{q}}^{(a)}\right)\right] ;
\end{aligned}
$$$$
S t_{e-I} f_{\vec{k}}^{(a)}+S t_{e-p h} f_{\vec{k}}^{(a)}=-\frac{e^{2}}{(2 \pi)^{3} \hbar} \int d \omega \delta\left(\hbar \omega-\varepsilon_{\vec{k}}-\varepsilon_{\vec{k}-\vec{q}}\right) \times
$$$$
\left.\times \int\left\{\left[f_{\vec{k}}^{(a)}\left(1-f_{\vec{k}-\vec{q}}^{(a)}\right)+f_{\vec{k}-\vec{q}}^{(a)}\right)\left(1-f_{\vec{k}}^{(a)}\right)\right)\right] \times
$$$$
\left.\left.\times \tanh \left(\frac{\hbar \omega}{2 k_{B} T}\right)+f_{\vec{k}}^{(a)}-f_{\vec{k}-\vec{q}}^{(a)}\right)\right\} \times
$$$$
\times\left[\left\langle\delta \varphi_{(I)}^{2}\right\rangle_{\omega, \vec{q}}+\left\langle\delta \varphi_{(p h)}^{2}\right\rangle_{\omega, \vec{q}}\right] d^{3} \vec{q} .
$$

Here

$\varepsilon_{\vec{k}}^{(a)}=\frac{\hbar^{2}}{2}\left(\frac{q_{x}^{2}}{m_{x x}^{(a)}}+\frac{q_{y}^{2}}{m_{y y}^{(a)}}+\frac{q_{z}^{2}}{m_{z z}^{(a)}}\right)$

is the dispersion law for $a$-carriers with ellipsoidal surfaces of constant energy (for corresponding suitable system of axes);

$$
\begin{aligned}
& \left\langle\varphi_{(I)}^{2}\right\rangle_{\vec{q}, \omega}=\frac{32 \pi^{3} e^{2} n_{I}}{\varepsilon_{L}^{2}\left[q^{2}+q_{0}^{2}(\vec{q})\right]^{2}} \delta(\omega) ; \\
& \left\langle\delta \varphi_{(p h)}^{2}\right\rangle_{\vec{q}, \omega}=\left[\Xi_{A}(\vec{q}) \frac{q^{2}}{q^{2}+q_{0}^{2}(\vec{q})}\right]^{2} \frac{2 \pi k_{B} T}{e^{2} \rho s^{2}} \delta(\omega) .
\end{aligned}
$$

Below, we will perform numerical calculations using this approximation:

$$
\Xi_{A}(q)=\Xi_{d}+(1 / 2) \Xi_{u} \text {. }
$$

Here (see, for example, Refs [5] and [6]), $\Xi_{d}$ and $\Xi_{u}$ are dilatation and shear deformation potential constants.

Designing the distribution function for equilibrium gas of $a$-carriers as $f^{0}\left(\varepsilon_{\vec{k}}^{(a)}\right)$, we write (see Refs [3,4]) $\Delta \varepsilon_{(a)}^{0}(\omega, \vec{q})=$

$$
=\frac{e^{2}}{\pi^{2} q^{2}} \int d^{3} \vec{k} \frac{f^{0}\left(\varepsilon_{\vec{k}}^{(a)}\right)-f^{0}\left(\varepsilon_{\vec{k}-\vec{q}}^{(a)}\right)}{\varepsilon_{\vec{k}-\vec{q}}^{(a)}-\varepsilon_{\vec{k}}^{(a)}+\hbar \omega+i 0} .
$$

\section{Then}

$$
\begin{aligned}
& q_{0}^{2}(\vec{q})=\frac{e^{2} m_{\perp} m_{\|}^{1 / 2} \sqrt{2 k_{B} T}}{\hbar^{3} \pi \varepsilon_{L}} \times \\
& \left.\times \sum_{a=1}^{M} \frac{1}{Q^{(a)}(\vec{q})} \int_{0}^{\infty} \frac{d \varsigma}{1+\exp \left(\varsigma-\eta_{a}\right)} \ln \left(\mid \frac{\sqrt{\varsigma}+Q^{(a)}(\vec{q})}{\sqrt{\varsigma}-Q^{(a)}(\vec{q})}\right)\right),
\end{aligned}
$$

where
$Q^{(a)}(\vec{q})=\frac{\hbar}{\sqrt{8 k_{B} T}}\left(\frac{q_{x}^{2}}{m_{x x}^{(a)}}+\frac{q_{y}^{2}}{m_{y y}^{(a)}}+\frac{q_{z}^{2}}{m_{z z}^{(a)}}\right)^{1 / 2}$.

For farther numerical calculations, we will simplify the form (9) to

$$
\begin{aligned}
& q_{0}^{2}(\vec{q})=q_{0}^{2}(q)=\frac{e^{2} M \sqrt{2 m_{\|} m_{\perp}^{2} k_{B} T}}{\pi \varepsilon_{L} \hbar^{3} \sqrt{w(q)}} \times \\
& \times \int_{0}^{\infty} \frac{d \gamma}{1+\exp (\gamma-\eta)} \ln \left(\left|\frac{\sqrt{\gamma}+\sqrt{w(q)}}{\sqrt{\gamma}-\sqrt{w(q)}}\right|\right) .
\end{aligned}
$$

Here

$$
\begin{aligned}
& \frac{1}{m^{*}}=\frac{1}{3}\left(\frac{2}{m_{\perp}}+\frac{1}{m_{\|}}\right)=\frac{2 L+1}{3 m_{\|}}, \\
& w(q)=\hbar^{2} q^{2} / 8 m^{*} k_{B} T, \quad \eta=\varepsilon_{F} / k_{B} T
\end{aligned}
$$

( $\varepsilon_{F}$ is the Fermi energy). The dimensionless energy $\eta$ is related with the concentration of mobile electrons $n$ by the relation

$$
n=\frac{M\left(2 \pi k_{B} T\right)^{3 / 2} m_{\perp} \sqrt{m_{\|}}}{2 \pi^{7 / 2} \hbar^{3}} \int_{0}^{\infty} \frac{w^{1 / 2} d w}{1+\exp (w-\eta)} .
$$

\section{Balance equation and kinetic coefficients}

Applying to both sides of Eq. (1) the operator $1 / 4 \pi^{3} \int \vec{k} d^{3} \vec{k}$

we obtain balance equations for forces:

$e \vec{E}+\vec{F}_{e-I}^{(a)}+\vec{F}_{e-p h}^{(a)}+\sum_{b=1}^{M} \vec{F}^{(a, b)}=0$

$(a=1,2, \ldots, M)$,

where

$\vec{F}_{e-I}^{(a)}+\vec{F}_{e-p h}^{(a)}=-\frac{e^{2}}{(2 \pi)^{6} n_{a}} \int d^{3} \vec{k} \times$

$\times \int \vec{q} d^{3} \vec{q} \int d \omega \delta\left(\hbar \omega-\varepsilon_{\vec{k}}^{(a)}+\varepsilon_{\vec{k}-\vec{q}}^{(a)}\right)\left\{f_{\vec{k}}^{(a)}-f_{\vec{k}-\vec{q}}^{(a)}+\right.$

$\left.+\left[f_{\vec{k}}^{(a)}\left(1-f_{\vec{k}-\vec{q}}^{(a)}\right)+f_{\vec{k}-\vec{q}}^{(a)}\left(1-f_{\vec{k}}^{(a)}\right)\right] \tanh \left(\hbar \omega / 2 k_{B} T\right)\right\} \times$

$\times\left[\left\langle\varphi_{(I)}^{2}\right\rangle_{\omega, \vec{q}}+\left\langle\varphi_{(p h)}^{2}\right\rangle_{\omega, \vec{q}}\right]$.

$\vec{F}^{(a, b)}=\frac{e^{4} \hbar}{4 \pi^{6} n^{(a)}} \int \vec{k} d^{3} \vec{k} \int d^{3} \vec{k}^{\prime} \times$

$\times \int d^{3} \vec{q} \frac{1}{q^{4}} \frac{\delta\left(\varepsilon_{\vec{k}}^{(a)}-\varepsilon_{\vec{k}-\vec{q}}^{(a)}-\varepsilon_{\overrightarrow{k^{\prime}}}^{(b)}+\varepsilon_{\vec{k}^{\prime}-\vec{q}^{\prime}}^{(b)}\right.}{\left|\varepsilon_{L}+\Delta \varepsilon(\omega=0, \vec{q})\right|^{2}} \mathrm{Y}_{a b}\left(\vec{k}, \vec{k}^{\prime}, \vec{q}\right) ;$

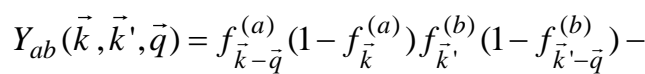

$-f_{\vec{k}}^{(a)}\left(1-f_{\vec{k}-\vec{q}}^{(a)}\right) f_{\vec{k}^{\prime}-\vec{q}^{\prime}}^{(b)}\left(1-f_{\vec{k}^{\prime}}^{(b)}\right)$. 
As an appropriate model of the non-equilibrium distribution function for carriers of $a$-group, we accept the Fermi function with the argument containing shift of velocity $\vec{v}^{(a)}(\vec{k})=\hbar^{-1}\left(\partial \varepsilon_{\vec{k}}^{(a)} / \partial \vec{k}\right)$ on the correspondent drift velocity $\vec{u}^{(a)}$ of whole $a$-group:

$$
f_{\vec{k}}^{(a)}=f^{0(a)}\left(\vec{v}^{(a)}(\vec{k})-\vec{u}^{(a)}\right) .
$$

Here $f^{0(a)}\left(\vec{v}^{(a)}(\vec{k})\right)$ is the equilibrium distribution function for $a$-carriers. obtain

Carrying out linearization of forces in Eq. (12), we

$$
\begin{aligned}
& \vec{F}_{e-I}^{(a)}+\vec{F}_{e-p h}^{(a)}=-e \tilde{\beta}^{(a)} \vec{u}^{(a)}, \\
& \vec{F}^{(a, b)}=-e \tilde{\xi}^{(a, b)}\left(\vec{u}^{(a)}-\vec{u}^{(b)}\right),
\end{aligned}
$$

where kinetic coefficients can be presented in the following forms:

$\beta_{\alpha \beta}^{(a)}=\frac{\hbar}{2(2 \pi)^{5} e n^{(a)} k_{B} T} \int d \omega \times$

$\times \int \frac{q^{2} d^{3} \vec{q}}{\sinh \left(\hbar \omega / k_{B} T\right)} q_{\alpha} q_{\beta} \operatorname{Im} \Delta \varepsilon_{(a)}^{0}(\omega, \vec{q}) \times$,

$\times\left[\left\langle\varphi_{(I)}^{2}\right\rangle_{\omega, \vec{q}}+\left\langle\varphi_{(p h)}^{2}\right\rangle_{\omega, \vec{q}}\right]$,

$\xi_{\alpha \beta}^{(a, b)}=\frac{\hbar^{2}\left(1-\delta_{a b}\right)}{2(2 \pi)^{4} e \varepsilon_{L}^{2} n^{(a)} k_{B} T} \int \frac{d \omega}{\sinh ^{2}\left(\hbar \omega / 2 k_{B} T\right)} \times$

$\times \int \frac{q^{4} q_{\alpha} q_{\beta} d^{3} \vec{q}}{\left[q^{2}+q_{0}^{2}(q)\right]^{2}} \operatorname{Im} \Delta \varepsilon_{(a)}^{0}(\omega, \vec{q}) \operatorname{Im} \Delta \varepsilon_{(b)}^{0}(\omega, \vec{q})$,

$n^{(a)}$ is the density of $a$-carriers; $\quad \alpha, \beta=x, y, z$.

From Eqs (12) and (16), one obtains the system of equations for drift velocities:

$$
\begin{aligned}
& \vec{E}-\tilde{\beta}^{(a)} \vec{u}^{(a)}-\sum_{b=1}^{M} \tilde{\xi}^{(a, b)}\left(\vec{u}^{(a)}-\vec{u}^{(b)}\right)=0 \\
& (a=1,2, \ldots, M) .
\end{aligned}
$$

Here, terms containing matrices $\tilde{\xi}^{(a, b)}$ respond for the intervalley drag. The value $\left(\tilde{\beta}^{(a)}\right)^{-1}$ is the mobility tensor for $a$-carriers, if one neglects the mentioned drag $\left(\tilde{\xi}^{(a, b)} \rightarrow 0\right)$.

\section{Mobility of band carriers in $n$-Si and $n$-Ge}

The structure of valleys in $n$-Si and $n$-Ge is shown in Fig. 1. At the beginning, let us consider the electron band in silicon.

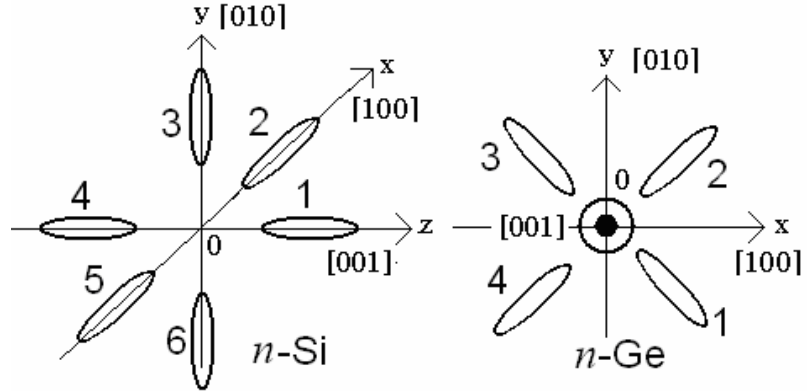

Fig. 1.

For its six valleys

$\tilde{\beta}^{(1)}=\tilde{\beta}^{(4)}=\left(\begin{array}{ccc}\beta_{\perp} & 0 & 0 \\ 0 & \beta_{\perp} & 0 \\ 0 & 0 & \beta_{\|}\end{array}\right) ; \tilde{\beta}^{(2)}=\tilde{\beta}^{(5)}=\left(\begin{array}{ccc}\beta \| & 0 & 0 \\ 0 & \beta_{\perp} & 0 \\ 0 & 0 & \beta_{\perp}\end{array}\right)$;

$\tilde{\beta}^{(3)}=\tilde{\beta}^{(6)}=\left(\begin{array}{ccc}\beta_{\perp} & 0 & 0 \\ 0 & \beta_{\|} & 0 \\ 0 & 0 & \beta_{\perp}\end{array}\right)$;

$\tilde{\xi}^{(1,2)}=\left(\begin{array}{ccc}\varsigma_{1} & 0 & 0 \\ 0 & \varsigma_{2} & 0 \\ 0 & 0 & \varsigma_{1}\end{array}\right)$

$\tilde{\xi}^{(1,3)}=\left(\begin{array}{ccc}\varsigma_{2} & 0 & 0 \\ 0 & \varsigma_{1} & 0 \\ 0 & 0 & \varsigma_{1}\end{array}\right) ; \quad \tilde{\xi}^{(2,3)}=\left(\begin{array}{ccc}\varsigma_{1} & 0 & 0 \\ 0 & \varsigma_{1} & 0 \\ 0 & 0 & \varsigma_{2}\end{array}\right)$.

Here

$\beta_{\|, \perp}=\frac{3 \hbar}{(2 \pi)^{5} \text { en } k_{B} T} \int d \omega \times$

$\times \int \frac{q^{2} q_{z, x}^{2} d^{3} \vec{q}}{\sinh \left(\hbar \omega / k_{B} T\right)} \operatorname{Im} \Delta \varepsilon_{(1)}^{0}(\omega, \vec{q}) \times$

$\times\left[\left\langle\delta \tilde{\varphi}_{(I)}^{2}\right\rangle_{\omega, \vec{q}}+\left\langle\delta \tilde{\varphi}_{(p h)}^{2}\right\rangle_{\omega, \vec{q}}\right] ;$

$\varsigma_{1,2}=\frac{3 \hbar^{2}}{(2 \pi)^{4} \varepsilon_{L}^{2} e n k_{B} T} \int \frac{d \omega}{\sinh ^{2}\left(\hbar \omega / 2 k_{B} T\right)} \times$

$\times \int \frac{q^{4} \operatorname{Im} \Delta \varepsilon_{(1)}^{0}(\omega, \vec{q}) \operatorname{Im} \Delta \varepsilon_{(2)}^{0}(\omega, \vec{q})}{\left[q^{2}+q_{0}^{2}(q)\right]^{2}} q_{x, y}^{2} d^{3} \vec{q}$.

A solution of the system (19) for $n$-Si can be represented in the form $\vec{u}^{(a)}=\tilde{\mu}^{(a)} \vec{E}$, where

$\tilde{\mu}^{(1,4)}=\left(\begin{array}{ccc}\mu_{\perp} & 0 & 0 \\ 0 & \mu_{\perp} & 0 \\ 0 & 0 & \mu_{\|}\end{array}\right) ; \tilde{\mu}^{(2,5)}=\left(\begin{array}{ccc}\mu_{\|} & 0 & 0 \\ 0 & \mu_{\perp} & 0 \\ 0 & 0 & \mu_{\perp}\end{array}\right)$;

$\tilde{\mu}^{(3,6)}=\left(\begin{array}{ccc}\mu_{\perp} & 0 & 0 \\ 0 & \mu_{\|} & 0 \\ 0 & 0 & \mu_{\perp}\end{array}\right)$ 
$\mu_{\perp}=\frac{\beta_{\|}+6 \zeta_{1}}{\beta_{\|} \beta_{\perp}+2\left(2 \beta_{\perp}+\beta_{\|}\right) \zeta_{1}} ;$

$\mu_{\|}=\frac{\beta_{\perp}+6 \zeta_{1}}{\beta_{\|} \beta_{\perp}+2\left(2 \beta_{\perp}+\beta_{\|}\right) \zeta_{1}}$.

The tensor of conductivity for totally mobile electrons:

$\sigma_{i j}=e \sum_{a=1}^{6} n^{(a)} \mu_{i j}^{(a)}=\delta_{i j} \sigma=\delta_{i j} e n \mu$,

$\mu_{(\mathrm{Si})}=\frac{1}{3} \frac{3+2\left(\beta_{\|}-\beta_{\perp}\right) /\left(\beta_{\perp}+6 \zeta_{1}\right)}{\beta_{\|}-4 \zeta_{1}\left(\beta_{\|}-\beta_{\perp}\right) /\left(\beta_{\perp}+6 \zeta_{1}\right)}=$

$=\frac{1+2 L}{3 \beta_{\|}} \frac{1+18 \varsigma_{1} L / \beta_{\|}(1+2 L)}{1+2(2+L) \varsigma_{1} / \beta_{\|}}$.

Note that the value $\varsigma_{2}$ does not enter to the formula (23); this value appears when galvano-magnetic effects are considered.

Analogous consideration for $n$-Ge gives a similar result:

$\mu_{(\mathrm{Ge})}=\frac{1+2 L}{3 \beta_{\|}} \frac{1+\left(12 \varsigma_{1} L / \beta_{\|}\right) /(1+2 L)}{1+(2+L)\left(4 \varsigma_{1} / 3 \beta_{\|}\right)}$.

One can see that the reason of intervalley drag (this effect is represented in formulae (23) and (24) by the value $\left.\zeta_{1}\right)$ is anisotropy of valleys $\left(\beta_{\|} \neq \beta_{\perp}\right.$, or $L \neq 1$ ).

We carry out farther calculations of the values $\beta_{\|}, \beta_{\perp}$ and $\zeta_{1}$ on the base of a simplifying procedure of preliminary partial average over angle expressions represented by three-dimensional integrals (see also Eqs (20), (21) and (10)). As a result, we obtain (here $n_{I}$ is the concentration of charged impurities):

$$
\begin{aligned}
& \beta_{\|}=L \beta_{\perp}, \quad \beta_{\|}=\beta_{\|}^{(I)}+\beta_{\|}^{(p h)} ; \\
& \beta_{\|}^{(I)}=\frac{16 e^{3} n_{I} m_{\|}^{2}}{\pi \hbar^{3} \varepsilon_{L}^{2} n(L+1)^{2}} \int_{0}^{\infty} \frac{w d w}{1+\exp (w-\eta)} \frac{1}{\left[w+w_{s c r}(w)\right]^{2}} ;
\end{aligned}
$$

$\beta_{\|}^{(p h)}=\frac{16\left[\Xi_{d}+(1 / 2) \Xi_{u}\right]^{2}\left(k_{B} T\right)^{3} m_{\|}^{4}}{\pi^{3} e \hbar^{7} \rho s^{2} n L^{2}} \times$

$\times \int_{0}^{\infty} \frac{w^{3} d w}{1+\exp (w-\eta)} \frac{1}{\left[w+w_{s c r}(w)\right]^{2}} ;$

$\varsigma_{1}=\frac{\sqrt{2} e^{3}\left(k_{B} T\right)^{3 / 2} \varpi}{\pi^{3} n \hbar^{6} \varepsilon_{L}^{2}}\left(\frac{3 m_{\|}}{2 L+1}\right)^{7 / 2} \times$

$\times \int_{0}^{\infty} \frac{\sqrt{w} d w}{[1+\exp (w-\eta)]^{2}} \frac{1}{\left[w+w_{s c r}(w)\right]^{2}} ;$
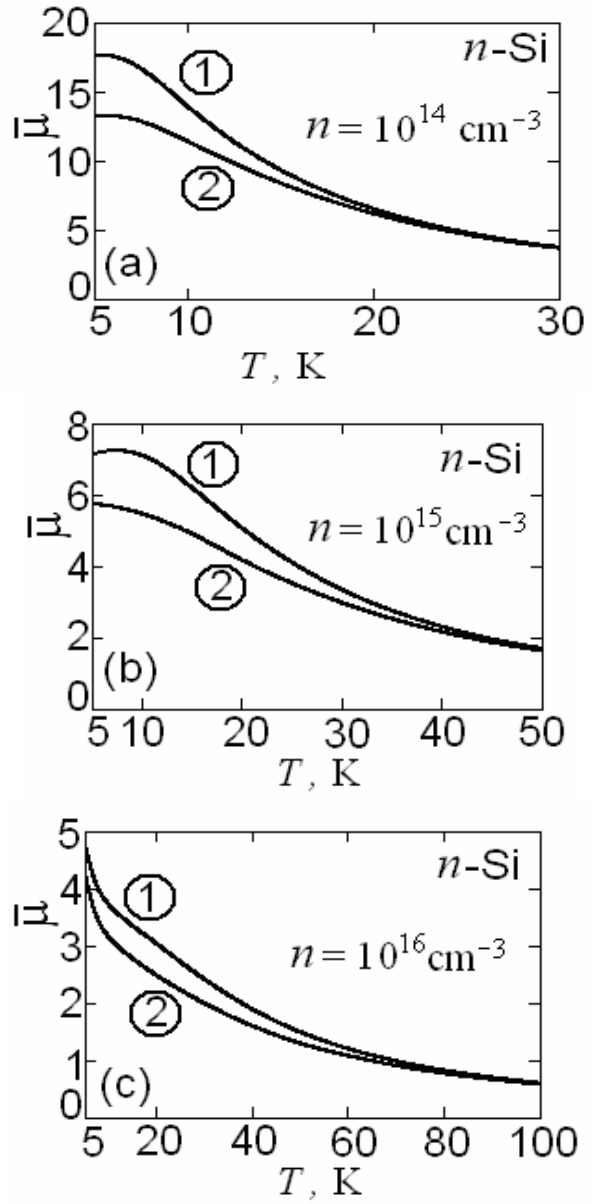

Fig. 2.

$w_{s c r}(w)=\frac{e^{2} M \sqrt{2 m_{\|}^{3}}}{8 \pi L \varepsilon_{L} \hbar m^{*} \sqrt{w k_{B} T}} \times$

$\times \int_{0}^{\infty} \frac{d \gamma}{1+\exp (\gamma-\eta)} \ln \left(\left|\frac{\sqrt{\gamma}+\sqrt{w}}{\sqrt{\gamma}-\sqrt{w}}\right|\right)$;

$\varpi=\int_{-\infty}^{\infty} \frac{\chi^{2} d \chi}{\sinh ^{2} \chi} \approx 3.29$.

Results of numerical calculations of the mobility at $n=n_{I}$ are presented for $n$-Si in Fig. 2, and for $n$-Ge in Fig. 3. Here,

$\bar{\mu}=\mu \times\left(e \tau_{0} / m_{0}\right)^{-1}$,

where $m_{0}=9.1066 \cdot 10^{-28} \mathrm{~g}, \tau_{0}=10^{-12} \mathrm{~s}$. In this case, $\bar{\mu}=1$ corresponds to $\mu=1.758 \cdot 10^{3} \mathrm{~cm}^{2} / \mathrm{V} \cdot \mathrm{s}$. Carrying out the calculations, we have accepted $M=6, L=4.8$, $\varepsilon_{L}=12, \quad\left(m_{\|} / m_{0}\right)=0.92, \quad \rho s^{2}=1.66 \cdot 10^{11} \mathrm{~Pa}$, $\Xi_{d}+(1 / 2) \Xi_{u}=-4.2 \mathrm{eV}$ for $n-\mathrm{Si}$, and $M=4$, $L=19.4, \varepsilon_{L}=16,\left(m_{\|} / m_{0}\right)=1.59, \rho s^{2}=1.26 \cdot 10^{11} \mathrm{~Pa}$, $\Xi_{d}+(1 / 2) \Xi_{u}=1.9 \mathrm{eV}$ for $n$-Ge. 

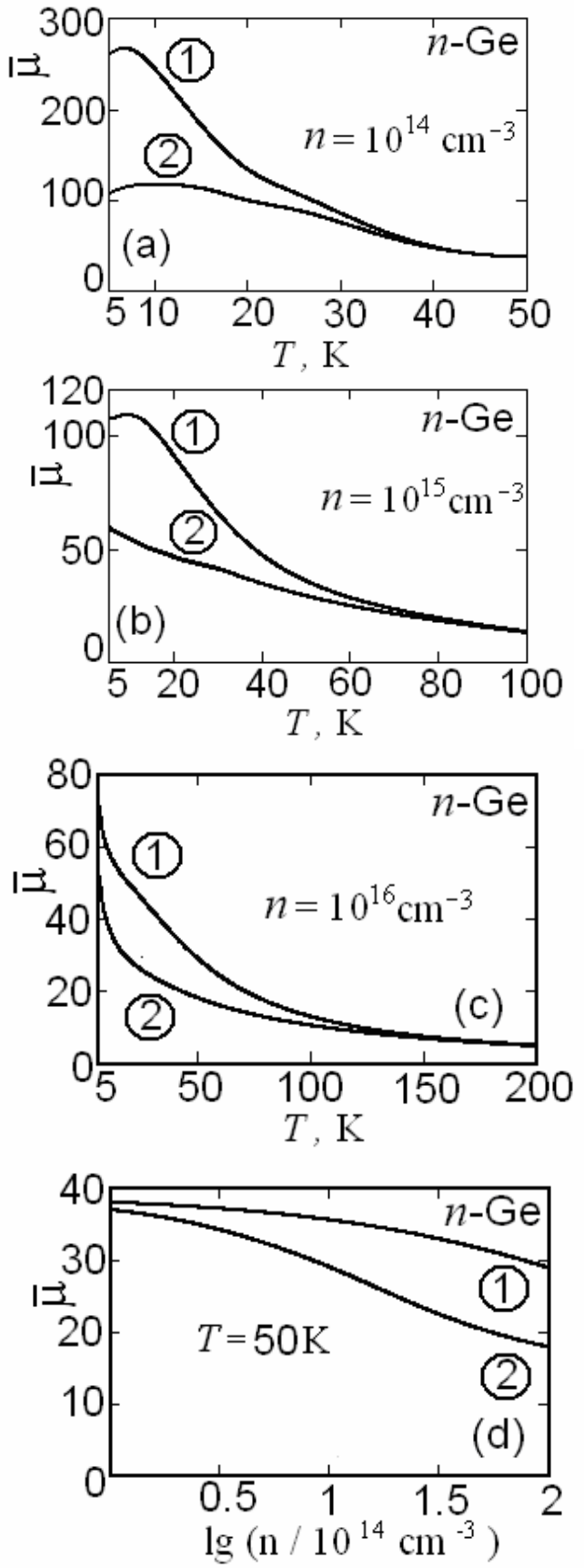

Fig. 3

Curves 1 in Figs 2 to 5 show the mobility of electrons calculated on the base of formulae (23) or (24) at $\varsigma_{1}=0$ (that is the case when the intervalley drag was not accepted in consideration). Curves 2 relate to the same formulae and represent the mobility with due regard for the intervalley drag $\left(\varsigma_{1} \neq 0\right)$. One can see from these figures that the intervalley drag in $n$-Si and $n$-Ge introduces an appreciable contribution to the mobility at sufficiently low temperatures. In germanium crystals, the considered effect manifests itself more clearly, because anisotropy of values in germanium considerably exceeds that in silicon.

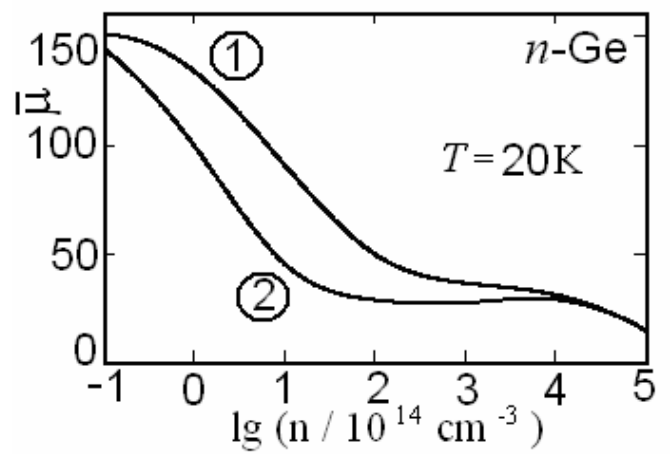

Fig. 4.
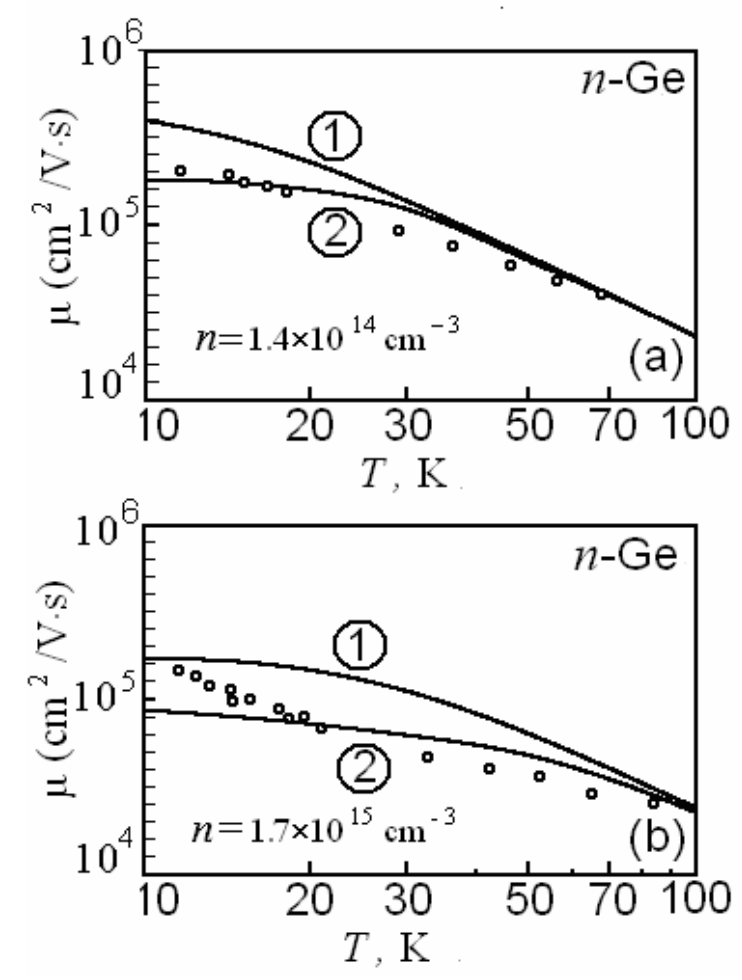

Fig. 5.

One can see from Fig. 4 that the intervalley drag practically disappears for high concentrations of carriers (in this case, the charge of every band carrier is substantially screened). This effect significantly weakens at a small concentration, because electron-electron scattering is rare and therefore unimportant.

Fig. 5 shows as calculated here curves as experimental data represented in Ref. [7]. The latter are shown by small circles. The author thinks that the curve 2 (intervalley drag is taken into consideration) better coordinates with experimental points than the curve 1 (intervalley drag is not taken into account). It was pointed out in Ref. [8] that the mobility in $n$-Ge calculated on the base of $\tau$-approximation substantially exceeds the experimentally measured one. 


\section{References}

1. F.G. Bass and Yu.G. Gurevich, Hot Electrons and Strong Electromagnetic Waves in Plasmas of Semiconductors and Gas Discharge. Nauka, Moscow, 1975 (in Russian).

2. F. Gantmacher and I.B. Levinson, Scattering of Current Carriers in Metals and Semiconductors. Nauka, Moscow, 1975 (in Russian).

3. E.M. Lifshits and L.P. Pitaevskiy, Physical Kinetics. Nauka, Moscow, 1979 (in Russian).
4. I.I. Boiko, Kinetics of Electron Gas Interacting with Fluctuating Potential. Naukova dumka, Kiev, 1993 (in Russian).

5. C. Herring, E. Vogt, Transport and deformationpotential theory for many-valley semiconductors with anisotropic scattering // Phys. Rev. 101, p. 944 (1956).

6. B.K. Ridly, Quantum Processes in Semiconductors. Clarendon Press, Oxford, 1982.

7. P.P. Debye, E.M. Conwell, Electrical properties of n-type germanium // Phys. Rev. 93, p. 696 (1954).

8. I.I. Boiko, To the theory of electron mobility in semiconductors // Fizika Tverd. Tela 1 (4), p. 574 (1959) (in Russian). 